\title{
Rechtsgeschichte
}

\section{Christian Windler}

Republik und Souveränität 
kaum mit den Bundeskompetenzen für Wasserbau und Forstpolizei verbunden und damit in eine Tradition des I9. Jahrhunderts gestellt werden. Die weittragende Bedeutung von Gewässerschutz und Raumplanung in der bundesgerichtlichen Rechtsprechung hätte in diesem Zusammenhang wenigstens erwähnt werden dürfen.

Insgesamt enthält das Buch - leider zu wenige - Ansätze für eine eigenständige Rechtsgeschichte der Schweiz; die könnte man vielleicht entlang der konsequenten Darstellung von Interdependenzen, Antagonismen und Eigentümlichkeiten schreiben (z. B. Föderalismus versus Zentralismus, Neutralität versus Europäisierung, direkte Demokratie und fehlende Verfassungs- gerichtsbarkeit) und mit der eingänglichen Beschreibung von Institutionen (Gerichtsorganisation, Anwaltsstand, Universitäten) verbinden. Es wäre auch angezeigt, sich von der Fixierung auf das "Verfassungsrechtliche", das "Politische " und die (vor dem I9. Jahrhundert ja doch ganz bescheidene) Gesetzgebung zu lösen und umgekehrt aus der Beschreibung von Gerichtsund Prozesswesen sich der - wenn es sie denn geben sollte - eigentlichen Rechtsgeschichte der Schweiz anzunähern. Insofern hat das Buch doch vor allem eine bibliografische Lücke gefüllt.

Matthias Schwaibold

\section{Republik und Souveränität*}

»Geburt der Republic «: Die zu besprechende Studie beinhaltet eine deutliche Absage an ein Verständnis von Republikanismus als einer zeitlosen Spezialität der Eidgenossenschaft seit den ersten Bünden. Maissen dekonstruiert in seiner Zürcher Habilitationsschrift diese Vorstellung, welche das schweizerische Selbstbewusstsein zutiefst geprägt und auch die Forschung stark beeinflusst hat. Dazu zeigt er die konkreten Umstände auf, unter denen die Kantone (»Orte«) erst im Laufe des I7. Jahrhunderts als » Republiken « bezeichnet wurden und unter denen sich ein explizit republikanisches Selbstverständnis entwickelte. Ziel ist »eine konsequente Historisierung von >Republik` und >Republikanismus`, die in der Schweizer Historiographie zu unreflektierten, aber wichtigen Konstanten der Interpretation geworden sind $«(33)$.
Maissen knüpft bei den Arbeiten von Karl Mommsen über Eidgenossen, Kaiser und Reich an, die etwa von Peter Moraw, Olaf Mörke, Marco Jorio und Bettina Braun weitergeführt worden sind. Im Laufe des I7. Jahrhunderts lösten sich die eidgenössischen Eliten vom Verständnis der Kantone als besonders privilegierten Gliedern des Heiligen Römischen Reiches - Quelle und Garant des in der Eidgenossenschaft geltenden Rechts - und übernahmen stattdessen republikanische Definitionen eigenstaatlicher Souveränität im Sinne von Jean Bodin. Eine deutliche Absage erteilt Maissen der Vorstellung eines spätmittelalterlichen und frühneuzeitlichen Kommunalismus als Grundlegung des republikanischen Staatsverständnisses in der Eidgenossenschaft, wie sie von Peter Blickle und dessen Schülern gepflegt wird: Der Republikbegriff entstamme

\footnotetext{
* Thomas Maissen, Die Geburt der Republic. Staatsverständnis und Repräsentation in der frühneuzeitlichen Eidgenossenschaft, Göttingen: Vandenhoeck \& Ruprecht 2006, 672 S., ISBN 3-525-36706-6
} 
nicht einer »kommunalistischen « Praxis, sondern sei von außen - aufgrund eines wohl kalkulierten Eigeninteresses vor allem von den diplomatischen Vertretern Frankreichs und der Niederlande - in die Eidgenossenschaft hineingetragen worden. Dort sei er in der Verbindung mit dem neuen Souveränitätskonzept zur ideellen Grundlage tendenziell bindungsfreier - »absoluter« - staatlicher Herrschaft geworden.

Maissen beginnt mit Analysen des Reichsgedankens bei Josias Simmler und der Souveränitätslehre Bodins sowie einer Untersuchung der Rolle der Freistaaten in der monarchischen Ordnung Europas. Quellennah zeigt er sodann, wie die eidgenössischen Eliten im Laufe des I7. Jahrhunderts die Sprache und die Repräsentationsformen eines souveränen Völkerrechtssubjektes erlernten. Als entscheidende Wende erscheinen die Verhandlungen in Münster und Osnabrück. Maissen vertieft und nuanciert die in jüngerer Zeit in Gang gekommene Revision älterer Interpretationen des Westfälischen Friedens als völkerrechtlicher Bestätigung eidgenössischer Souveränität. Er zeigt, wie sich der Basler Bürgermeister Wettstein nach Westfalen begab, um eine Bestätigung und Ausweitung der reichsrechtlichen Privilegien zu erhalten. Im Zuge der Verhandlungen bewogen die französischen und niederländischen Gesandten Wettstein dazu, sich stattdessen auf die tatsächlich ausgeübte Souveränität zu berufen. Dennoch wurde in den Friedensverträgen mit der »exemptio « eine reichsrechtliche Lösung und nicht die eigenstaatliche Souveränität festgeschrieben. Während Wettstein die Exemption zur reichsrechtlichen Anerkennung einer bereits bestehenden Souveränität umdeutete, wollten nicht nur der Kaiser und die Reichsstände, sondern auch viele Zeitgenossen in der Eidgenossenschaft darin keine definitive Trennung vom Reichsverband sehen.
Entschieden neue Erkenntnisse bringt die Analyse der Veränderungen des Staatsverständnisses nach I648: Damals verband sich der Souveränitätsbegriff mit einem Republikbegriff, der die kollektive Variante des frühneuzeitlichen Obrigkeitsstaates bezeichnete. Im Anschluss an Quentin Skinner berücksichtigt Maissen eine Vielzahl verschiedener Quellen (Bildquellen ebenso wie Schriftquellen). Er weist auf diese Weise nach, wie sich um den Republikbegriff herum eine Reihe weiterer neuer Leitbegriffe durchsetzte, gleichzeitig mit der neuen politischen Sprache aber auch eine neue Ikonographie - etwa die Landespersonifikation der »Helvetia « - entstand.

$\mathrm{Zu}$ den neuen Leitbegriffen gehörte insbesondere jener der Neutralität. In der Form des »Stillesitzens « hatte die Zurückhaltung einzelner Bundesglieder (Basel, Schaffhausen) in den internen Konflikten zwar bereits seit der Zeit um I $500 \mathrm{zu}$ den explizit eingeforderten Bundespflichten gehört. Ebenso hatten die Dreizehn Orte gelernt, dass die Zurückhaltung in den Konflikten zwischen den europäischen Mächten angesichts der inneren Gegensätze für den Bundeszusammenhalt unabdingbar war. Zum Ausdruck höchster politischer Tugend wurde die Neutralität indessen erst seit der zweiten Hälfte des I7. Jahrhunderts und nicht etwa im Gefolge der Niederlage von Marignano I 5 I 5 , wie dies die ältere Historiographie behauptet hat. Wenn sich Maissen der Stellung der eidgenössischen Orte in den europäischen Mächtebeziehungen des I7. und I 8 . Jahrhunderts zuwendet, kann er einen tief greifenden Wandel auch in der Fremdwahrnehmung feststellen. Die Veränderungen betrafen ebenso die Praxis des diplomatischen Zeremoniells wie auch die Reichspublizistik, wobei es den Kantonen allerdings schwer fiel, als Republiken in der monar- 
chisch dominierten Staatenwelt Europas zu bestehen.

Den heterogenen bündischen Strukturen entsprechend verlief der Veränderungsprozess in den eidgenössischen Orten jeweils in unterschiedlichen Bahnen. In den katholischen Orten der Innerschweiz blieb der Bezug auf Kaiser und Reich wesentlich länger präsent als in Zürich oder Bern. Diese innereidgenössischen Unterschiede spiegelt Maissen am besonders detailreich untersuchten reformierten Zürich, dessen Schlüsselrolle bei der Formierung der neuen politischen Sprache er überzeugend nachweist. Die Zürcher standen in engem Kontakt mit den Niederländern, welche ihre schweizerischen Glaubensbrüder zum Rückzug der Soldtruppen aus Frankreich bewegen wollten und ihnen zugleich die neue republikanische Sprache vermittelten. In der Stadt an der Limmat entstanden sodann besonders früh aufklärerische Sozietäten (insbesondere das I679 gegründete Collegium Insulanum), in denen das herrschaftliche Verständnis von Republik debattiert wurde. Bereits in der Bürgerbewegung von I7I3 manifestierten sich auch die Widersprüche zwischen naturrechtlich begründeter Freiheit und der Praxis des Stadtregiments.

Mit einem Ausblick auf die helvetische Republik grenzt Maissen abschließend die frühneuzeitliche »Republic" als souveräne, tendenziell absolute Kollektivherrschaft noch einmal entschieden vom emanzipatorischen Republikanis- mus der Sattelzeit ab. Die Opposition gegen die Einengung der politischen Partizipation auf einen kleinen Kreis regimentsfähiger Familien hatte indessen in der Polysemie der »freien Republic « wirksame Anknüpfungspunkte gefunden.

Die sorgfältig recherchierte Habilitationsschrift wirft in vielerlei Hinsicht neues Licht auf das Staatsverständnis der frühneuzeitlichen Eidgenossenschaft. Quellennah untersucht Maissen das politische Selbstverständnis und die Selbstdarstellung der eidgenössischen Eliten, deren politisches Denken und Schreiben, Sprechen und Argumentieren. Insgesamt zeugt die Studie von profunder Kenntnis der politischen Sprache der Frühen Neuzeit. Umso mehr überrascht daher die Behauptung, das Wort »Absolutismus « sei »im I7. Jahrhundert zu einem umstrittenen Leit- und Kampfwort « geworden (39), dürfte doch inzwischen hinlänglich bekannt sein, dass es sich dabei nicht um einen zeitgenössischen Begriff handelt.

Maissen provoziert; es bleibt zu hoffen, dass die Herausforderung aufgenommen wird. Dies betrifft insbesondere die aufgrund der Untersuchungsanlage nur am Rande gestellte Frage nach dem Zusammenhang zwischen der sozialen Praxis von Herrschaft und deren in der neuen politischen Sprache postulierten tendenziellen Bindungsfreiheit.

\section{Christian Windler}

